Full length article

\title{
Novel atomic-scale mechanism of incipient plasticity in a chemically complex CrCoNi medium-entropy alloy associated with inhomogeneity in local chemical environment
}

\author{
Fu-Hua Cao ${ }^{\mathrm{a}, \mathrm{b}, \mathrm{c}}$, Yun-Jiang Wang, ${ }^{\mathrm{a}, \mathrm{b}, \mathrm{c}, *}$, Lan-Hong Dai ${ }^{\mathrm{a}, \mathrm{b}, \mathrm{c}, *}$ \\ a State Key Laboratory of Nonlinear Mechanics, Institute of Mechanics, Chinese Academy of Sciences (CAS), Beijing 100190, China \\ ${ }^{\mathrm{b}}$ School of Engineering Science, University of Chinese Academy of Sciences, Beijing 101408, China \\ ${ }^{\mathrm{c}}$ CAS Center for Excellence in Complex System Mechanics, Beijing 100190, China
}

\section{A R T I C L E I N F O}

\section{Article History:}

Received 19 March 2020

Revised 12 May 2020

Accepted 14 May 2020

Available online 22 May 2020

\section{Keywords:}

Dislocation nucleation

Medium-entropy alloy

Local chemical order

Molecular dynamics

Density functional theory

\begin{abstract}
A B S T R A C T
Understanding the incipient plastic mechanism in metals is critical for their associated mechanical properties. While heterogeneous dislocation nucleation from pre-existing defects constitutes the most prevalent onset mechanism of plasticity in the conventional solutions, such a scenario may break down in the recently emerging chemically-disordered high/medium entropy alloys (HEAs/MEAs), owing to their unique multiplecomponent feature and the inevitable inhomogeneity in local atomic environments. Here, classical molecular dynamics simulations and first-principles density functional theory calculations are carried out to study the atomic-scale mechanisms governing the incipient plasticity in a prototypical chemically complex face-centered cubic (fcc) CrCoNi MEA. Dislocation nucleation is found to occur preferentially at an energetically unstable defect cluster with body-centered cubic like (bcc-like) atomic environment as a precursor, after certain deformation before plasticity, which is in contrast with the usual mechanism of heterogeneous dislocation nucleation in the conventional solute solution metals. The minimum energy pathway of dislocation nucleation from a bcc precursor is discussed to rationalize the usual phenomenon. First-principles athermal quasi-static compression test validates the mechanism suggested by atomistic simulations. Further electronic structure analysis suggests that the local bcc-like defect cluster is related to the localized electronic behaviors of $\mathrm{Cr}$ atoms and the weak $\mathrm{Cr}-\mathrm{Cr}$ bonding, which promote the dislocation nucleation and therefore the incipient plasticity of $\mathrm{CrCoNi}$ MEA. The atomic and electronic insights reported here highlight the significant role of local chemical order in determining the mechanical property, and shed light on the strategy of optimizing mechanical performance via tailoring composition and local atomic arrangement in the generic highly concentrated solutions.
\end{abstract}

(c) 2020 Acta Materialia Inc. Published by Elsevier Ltd. All rights reserved.

\section{Introduction}

The high-entropy alloys (HEAs) or complex concentrated alloys (CCAs), and their derivatives (e.g., medium- entropy alloys, MEAs), in which multiple principal elements in equal or near equal molar ratio are occupied randomly on the topologically ordered crystallographic lattices with a high chemically disorder, have emerged as an exciting new class of metallic structural materials [1-9]. This unique atompacking structure imparts HEAs many extraordinary mechanical properties, such as exceptional fracture toughness [10-14], great potential to overcome traditional strength-ductility trade-off [15-24], superior “self-sharpening” capability [25], outstanding

\footnotetext{
* Corresponding author at: State Key Laboratory of Nonlinear Mechanics, Institute of Mechanics, Chinese Academy of Sciences (CAS), Beijing 100190, China.

E-mail addresses: yjwang@imech.ac.cn (Y.-J. Wang), Ihdai@Inm.imech.ac.cn (L.-H. Dai).
}

resistance to hydrogen embrittlement [26-29], impact resistance [30-32], and radiation resistance [33-35] etc. The special structure features i.e. lattice distortion caused by atomic mismatch and chemical disordering are often invoked as the reason for their excellent properties [36, 37]. Actually, the lattice distortion often intertwine with the chemical effect from electronic interaction and necessitate inhomogeneous distribution of the elements, where there will be local preference (or avoidance) for bonding between certain nearestneighbor species $[7,38]$. This composition inhomogeneity is universal in HEAs and has been detected in pioneering CrMnFeCoNi and derivative $\mathrm{CrFeCoNiPd}$ HEA [7, 39, 40]. The inhomogeneity imposes a rugged atomic and energy landscape for plastic deformation, leading to ongoing change in the local mechanical conditions for the deformation evolution [7].

The role of the composition inhomogeneity in the deformation of HEAs/MEAs has been increasingly studied in term of local chemical order (LCO), which has been verified in recent experiments [41, 42] 
and atomic modeling [39, 43-45]. Li et al. [44] recently constructed a reasonable atomic interaction potential for $\mathrm{CrCoNi}$ MEA and made MD simulations using this potential as a standard A-B-C complex alloy model. They demonstrated that the LCO heightens the ruggedness of the local energy landscape and raises the activation barriers that govern dislocation activities for CrCoNi MEA. By employing DFT calculations, Ding et al. [46] showed that the SFEs and the energy difference between the face-centered cubic (fcc) and hexagonal closepacked (hcp) phases of CrCoNi MEA can be tailored over a wide range of values by tuning its LCO. Most recently, Chen et al. [40] found that the variations in local chemical composition of CrMnFeCoNi HEA promote a high density of Lomer-Cottrell locks, which facilitate the construction of the stacking-fault networks to provide nucleation sites for the hcp phase transformation. The unusual dislocation behavior in HEAs caused by their composition inhomogeneity, as systematic elucidated by Ma [7], may further enhance our understanding of the intrinsic difference for deformation between HEAs and the traditional metals since the stacking fault (SF), twinning, and phase transition are actually partial-dislocation mediated process [7, 40, 47-49]. The increasing successes in the understanding of the coupling effect of local chemical bond to defect behaviors facilitates the development of a more-general theoretical approach of predicting the deformation process.

However, a specific issue remaining to be resolved pertaining to how does the local chemical environment affect the dislocation nucleation. Such information is essential to enhance our understanding of fundamental deformation mechanism of these alloys. For traditional alloys, it has been demonstrated that the rapid drop of dislocation nucleation energy barrier with temperature increasing is attributed to the weakening of atomic bonds caused by thermal expansion [50], which would be the origin for the well-defined thermally activated nature of dislocation nucleation [50-54]. This implies that the nature of chemical bonds play an important role in dislocation nucleation, which would become more prominent and intricate for HEAs/MEAs due to their random distribution of elements which would experience complex displacement processes during deformation. For example, Sharma et al. [55] have revealed a specific atomic rearrangement with the increase in unlike $\mathrm{Al}-\mathrm{Co}$ and $\mathrm{Al}-\mathrm{Cr}$ pairs and decrease in like $\mathrm{Al}-\mathrm{Al}$ pair at high strain during the compression of a AlCrCoFeNi HEA. Therefore, there is an urgent demand for unraveling the underlying physical picture of the self-organized atomic rearrangement during dislocation nucleation to clarify the influence mechanism of local chemical bonds. Present experimental advances are still extremely difficult to catch the atomic mechanism of dislocation nucleation involving the change of local chemical bonds [53, 56]. Atomistic simulations can provide a real-time and atomic-scale monitoring of the deformation processes such as dislocation nucleation, motion and reactions [44, 52, 57-59]. An electronic-level description of atomic bonding is also required to understand related physical mechanisms, which can be provided by ab initio quantum mechanical calculations performed within the DFT. Accordingly, we propose to couple the MD simulations with the first-principles DFT calculations in this paper, to elucidate the atomic-level origin and pathway of dislocation nucleation and their intrinsic link with the LCO in chemically complex CrCoNi MEAs.

\section{Methods}

\subsection{MD simulations}

MD simulations were performed using the Large-scale Atomic/ Molecular Massively Parallel Simulator (LAMMPS) package [60], where the recently developed DFT-calibrated embedded-atom potential for CrCoNi MEAs by Li, Sheng, and Ma [44] is used. This potential provides a relatively accurate description of both the unstable and stable SFEs compared with DFT calculations, which is important for atomic simulations of dislocation nucleation [44]. A 3D periodic computational cell is used to investigate the dislocation nucleation in single crystalline CrCoNi MEA under uniaxial compression at different strain rates of $1.0 \times 10^{8}, 1.0 \times 10^{9}, 5.0 \times 10^{9}$ $1.0 \times 10^{10}$ and $2.5 \times 10^{10} \mathrm{~s}^{-1}$. The simulation CrCoNi single crystal block ( $\sim 860,000$ atoms) was generated with a cell size of $24.41 \mathrm{~nm}$ along the crystallographic directions of [100], [010] and [001] respectively. The average lattice parameter of this alloy is $3.559 \AA$. To create the MEA with traditional random solid solution (RSS) structures, $\mathrm{Ni}$ atoms in fcc phase were randomly selected and replaced with $\mathrm{Co}$ and $\mathrm{Cr}$ until the desired composition was achieved. RSS samples with different compositions, including equiatomic $\mathrm{CrCoNi}$ (medium $\mathrm{Cr}$ ), non-equiatomic $\mathrm{Cr}_{44} \mathrm{Co}_{28} \mathrm{Ni}_{28}$ (high $\mathrm{Cr}$ ) and $\mathrm{Cr}_{22} \mathrm{CO}_{39} \mathrm{Ni}_{39}$ (low $\mathrm{Cr}$ ), are used to study the effect of $\mathrm{Cr}$ content on the mechanical behaviors of $\mathrm{CrCoNi}$ alloys. Partially ordered equiatomic MEA samples with $\mathrm{L}_{2} \mathrm{Cr}$ structure are created by fixing $\mathrm{Cr}$ element in the cubic corner sites, while the face-centered sites are randomly occupied by the other two elements ( $\sim 33.3 \%$ for $\mathrm{Co}$ and $\mathrm{Ni}$, respectively) and the remaining $\mathrm{Cr}(\sim 8.33 \%)$.The same procedure is used for creating $\mathrm{L} 1_{2} \mathrm{Co}$ and $\mathrm{L} 1_{2} \mathrm{Ni}$ equiatomic MEA samples. For the absolute RSS sample, the atoms are equivalent and randomly distributed in the lattices. However, for the alloyed $\mathrm{L} 1_{2} \mathrm{X}$ structure, the $\mathrm{X}$ ( $\mathrm{Cr}, \mathrm{Co}$ or $\mathrm{Ni}$ ) atoms can only occupy the second nearest neighbor shell and, thus, possess fewer $\mathrm{X}-\mathrm{X}$ pairs.

Before uniaxial compression along [001] orientation, where the normal stresses in the lateral directions vanish, all the configurations are thoroughly relaxed with energy minimization and then being equilibrated in a isobaric-isothermal (NPT) ensemble [61] at a pressure of zero and a temperature of $300 \mathrm{~K}$ for $10 \mathrm{ps}$. After the relaxations, the uniaxial compressive loading was applied to the MEA samples at room temperature $(300 \mathrm{~K})$ and at a constant strain rate. The compressive strain at each time step along the [001] orientation was uniformly increased by $\Delta \varepsilon=\dot{\varepsilon} \Delta t$, where $\Delta t$ was the MD time step of 1 fs and $\dot{\varepsilon}$ was the loading strain rate $\left(1.0 \times 10^{8}-2.5 \times 10^{10}\right.$ $\left.s^{-1}\right)$. The visualization of the atomic configurations was performed using the Polyhedral Template Matching (PTM) method as described in Ref. [62] and has been implemented in the OVITO software [63].

The free-end nudged elastic band (FENEB) method $[64,65]$ is used to search the minimum energy path (MEP) of dislocation nucleation under a given uniaxial stress. The fully relaxed configuration with prescribed strain is chosen as the initial state. A configuration with the same axial strain but contains a dislocation loop is set for final state. FENEB calculations were performed with 12 system replicas (including the initial and final states) with a force tolerance convergence criterion of $0.005(\mathrm{eV} / \mathrm{A})$. The spring constant in the FENEB method is set to be $0.1 \mathrm{eV} / \AA^{2}$.

\subsection{First-principles calculations}

To further verify the structural evolution and the corresponding electronic origin during uniaxial compression of CrCoNi MEAs, DFT based compression calculation was carried out, using a plane wave method, as implemented in the Vienna Ab initio Simulation Package (VASP) [66]. A category of $4 \times 6 \times 4$ supercells with special quasi random structures (SQS), which closely mimic the most relevant nearest neighbor pair and multisite correlation functions of random alloy in small systems with periodic boundary conditions, were generated through a were generated through a Monte Carlo (MC) algorithm within the Alloy Theoretic Automated Toolkit (ATAT) package, developed by Alex van de Walle et al. [67]. The objective function need to be minimized is $\mathrm{Q}=-\mathrm{wL}+\sum_{\alpha \in A}\left|\Delta \rho_{a}(\sigma)\right|$, where $\Delta \rho_{a}(\sigma)$ denotes the gap of correlation function between a candidate state and fully disordered state. The best SQS supercells were chosen by running separate MC simulations with different seeds, where the configuration with the lowest objective function was adopted to further calculation. 
A gradient-corrected functional in the Perdew-Burke-Ernzerhof (PBE) form was used to describe the exchange and correlation interactions in DFT [68]. Electron-ion interactions were treated within the projector-augmented-wave (PAW) method [69]. A sufficiently high energy cutoff of $400 \mathrm{eV}$ was adopted for the plane-wave basis set expansion. The energy convergence criterion was set to be $10^{-6} \mathrm{eV}$. Brillouin zone integrations were performed using $3 \times 1 \times 3$ Monkhorst-Pack k-point grids. Collinear spin polarization (ISPIN $=2$ ) is enabled in all the calculations. The stress-strain relations of the CrCoNi MEAs under uniaxial compression are determined using a method described previously by Roundy et al. [70]. The lattice vectors are deformed incrementally in the $\langle 001\rangle$ direction. At each deformation step, the structure and atoms are simultaneously relaxed such that the relaxed stresses, except for the fixed direction ( $z$ axis), are smaller than $0.015 \mathrm{GPa}$, and the forces acting on each atom is below $0.01 \mathrm{eV} / \mathrm{A}$.

\section{Results and discussions}

\subsection{Mechanical response and deformation mechanism}

In order to analyze the mechanical and structure response, we will track the stress and structural evolution at strain rate of $1.0 \times 10^{8} \mathrm{~s}^{-1}$ which is typical of MD simulations. The result are shown in Fig. 1, in which the dependence of the numbers of bcc and hcp atoms on the compressive strain (the right axis) are also illustrated. In the linear elastic region, the atoms all keep their original lattice structure of fcc type. When the strain reaches the critical of $\sim 5 \%$, some atoms deviate from their equilibrium positions partially and rearrange into a bcc structure with a lower coordination number. These bcc atoms break the local symmetry and could be considered as a structural defect, leading to the slope decrease in nonlinear elastic region. In this sense, the $f c c$-to-bcc transition yields kind of pseudo plastic behavior. When stress increases to a critical value of $\sim 4.4 \mathrm{GPa}$, a significant stress drop occurs in the stress-strain curves, signifying the onset of plastic deformation. The moment of the onset of plasticity can be regarded as the beginning of the formation of dislocation loop bounding a stacking fault region, which is reflected at the beginning of the intensive growth of the number of hcp atoms at strain of $6 \%$ (indicated by the first vertical dash line in Fig. 1). When the nucleation process finishes, the dislocation loop propagates quickly accompanied by the dramatically increase of the number of hcp atoms,

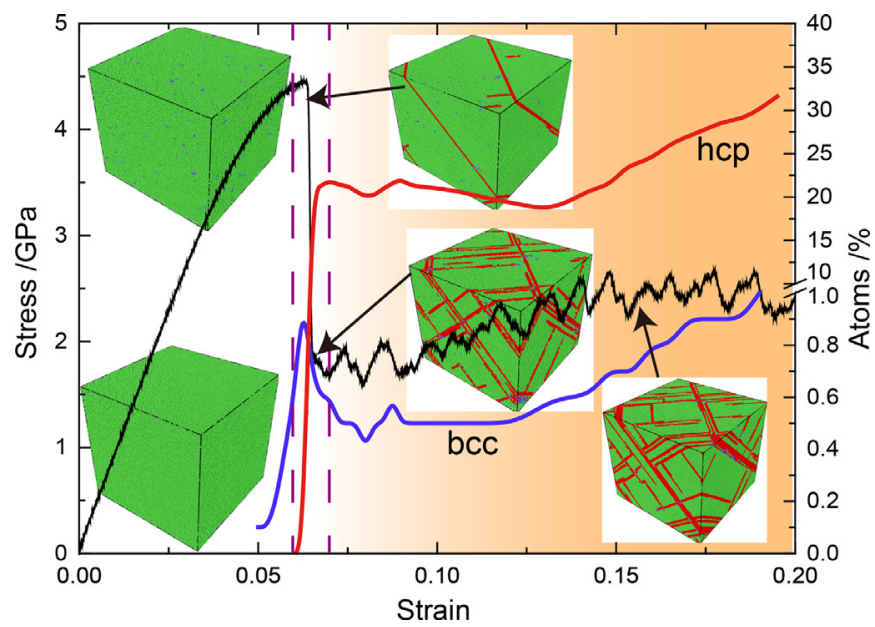

Fig. 1. The evolution of stress and structure as a function of strain for the RSS CrCoN MEA under strain rate of $1.0 \times 10^{8} \mathrm{~s}^{-1}$. Atoms colored by blue, red and green represent those in bcc, hcp and fcc structures, respectively. The hcp atoms are actually those in stacking fault regions after the leading partial dislocations. (For interpretation of the references to colour in this figure legend, the reader is referred to the web version of this article.) which leads to a significant stress drop in a sudden manner. Interestingly, the stress drops more slowly with a finite slope at relatively higher strain rates $\left(>10^{9} \mathrm{~s}^{-1}\right)$ after yielding (See Fig. S1 in the Supplemental Materials). In this case, the number of hcp atoms increases and stress decreases in a mild manner, eventually forming abundant small SFs (see Fig.S2 in the Supplemental Materials). These small SFs intersect with each other and finally form a dense 3D SFs network, hindering the further growth of small loops and thus delay the stress drop.

\subsection{Atomic and physical mechanism of dislocation nucleation}

To reveal the incipient process of dislocation nucleation, the atomic scale structural evolution of the dislocation enclosed SFs is traced. The results are shown in Fig. 2. Here, only three layers of atoms at a (111) slip plane are plotted. For a more intuitive display of the stacking order, the radius of the $\mathrm{Ni}, \mathrm{Co}$ and $\mathrm{Cr}$ atoms are set to be the same. In Fig. 2, from left to right, the common neighbor analysis (CNA) is used to indicate the lattice symmetry, which can distinguish whether an atom is in fcc, bcc, hcp (hexagonal cubic phase), or other disordered phases. The centro-symmetry parameter (CSP) is also displayed to measure the local lattice distortion around an atom and, it can be used to characterize whether the atom belongs to a perfect lattice, or resides in a SF. The displacement vector and non-affine squared displacements [71], $D_{\min }^{2}$, are also shown to trace the change in atom positions, which can detail the motion pattern of atoms during dislocation nucleation.

Although a few atoms rearrange to a bcc-like environment at $\varepsilon=0.0634$, the atoms still in a close packed form as such in the perfect fcc lattice (Fig. 2a1). Note that not only the bcc-like defect atoms but also some fcc atoms possess a high value of CSP (Fig. 2a2), indicating a strong lattice distortion effect of the chemically complex $\mathrm{CrCoNi}$ MEA. This distortion is consisted as the static disorder due to atom radius mismatch and the dynamic disorder due to thermal vibrations constraint in different local chemical environments. The disordered distribution of atomic displacement relative to the initial undeformed configuration $(\varepsilon=0)$ indicates that there is no preferred direction of atomic movement in the deformation process, at least before the moment of $\varepsilon=0.0634$ (Fig. 2a3 and a4). With increasing strain up to 0.0635 , more fcc atoms rearrange to be bcc-like defects and form a successive double-layer nanoscale defect cluster (see Fig. 2b1), accompanied by random atomic movements (see Fig. 2b3). Similar defect clusters are reported in a Al-Cu alloy [72]. This double-layer defect cluster further expands with the strain increasing to 0.0636, at which some central-region bcc atoms rearrange to hcp severing as nucleus for SF (the red atoms in Fig. 2 c1). The obvious split within this double-layer defect cluster indicates that the stacking order in this region is converted from $A B C$ to $A B A$, in agreement with the stacking sequence of a SF in a fcc lattice (Fig. 2c1). Here A, B, and C denote three nonequivalent atomic layers with different stacking positions in a fcc lattice. The large nonaffine squared displacements from $\varepsilon=0.0635$ to 0.0636 indicates a strong nonaffine effect of atomic motion during dislocation nucleation (Fig. 2c4). Meanwhile, the displacement vectors of the hcp atoms in the adjacent layer are arranged in a certain direction, suggesting that such nonaffine deformation process is caused by the relative interlayer slip (Fig. 2c3). The significant difference of the $D_{\min }^{2}$ between these adjacent layer atoms also confirms the interlayer slip during formation of a SF (Fig. 2c4). A big SF enclosed with a partial dislocation loop (green loop as show in the inset in d1) nucleates and expands quickly based on this hcp embryo when the strain increases to 0.067 (Fig. $2 \mathrm{~d} 1$ and $\mathrm{d} 2$ ). This nucleation process is governed by the atom shuffling mechanism, in which a majority of the atoms are shifted along [101] crystallographic orientation and a few atoms move along other orientations (Fig. 2d3). Note that the atoms in the center of SF (white circle, Fig. 2d4) is of high value of CSP (Fig. 3c2), but small $D_{\min }^{2}$. At this moment, the SF embryo 

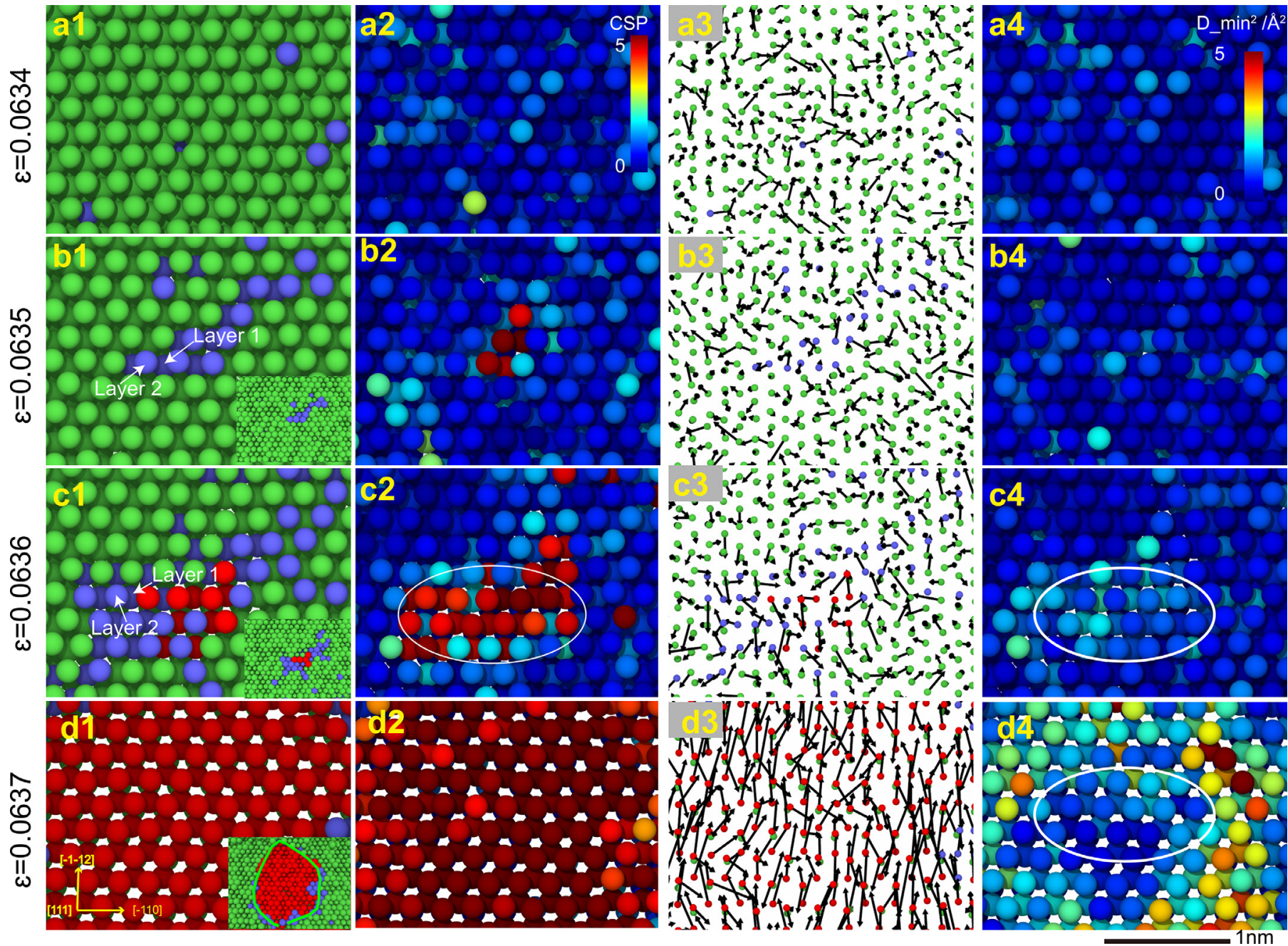

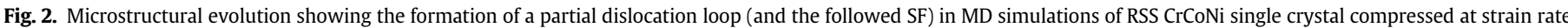

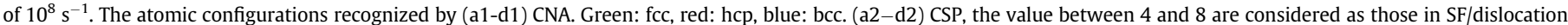

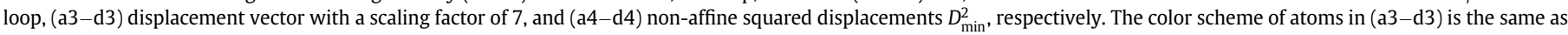

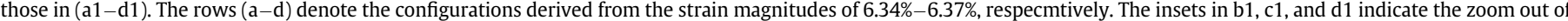
the dislocation nucleation regions. (For interpretation of the references to colour in this figure legend, the reader is referred to the web version of this article.)

has already formed, and the boundary atoms on the front of dislocation loop bear most of nonaffine distortions. This further confirms that the double-layer defect cluster severs as the embryo of SF and the formation of this embryo dominates the nucleation of dislocation.

To gain a deeper understanding of the nucleation mechanisms, we calculate the stress dependence of the activation energy of the dislocation nucleation, using the FENEB method. The activation energies and the corresponding minimum energy path (MEP) at $0 \mathrm{~K}$ are plotted in Fig. 3. A noteworthy feature is that the trend of the activation energy can be divided into two different regimes and can be fitted by different empirical equations. It signifies different physical characteristics of dislocation nucleation at low and high stress condition, as shown in Fig. 3a. As shown, the activation energy decreases with stress in the full stress range. This is understood since mechanical work reduces the barrier height and facilitate the nucleation. We fit the activation energy versus stress curves with the well-known empirical Kock's law in form of $Q(\sigma)=A\left(1-\left(\frac{\sigma}{\sigma_{0}}\right)^{\alpha}\right)$, here $\sigma_{0}$ is an athermal stress, and $A, \alpha$, and $\beta$ are fitting parameters [73] . For $\sigma \leq$ $4.25 \mathrm{GPa}$, the activation energy decreases with stress with exponents of $\alpha=0.51$ and $\beta=1.21$. However, at high stress $\sigma \geq 4.25 \mathrm{GPa}$, it reads $\alpha=1$ and $\beta=0.766$. The activation volume $\Omega$ is defined as the negative derivative of activation energy with respect to stress, i.e.,
$\Omega=-\frac{\partial Q}{\partial \sigma}$. Activation volume is usually used as a kinetic signature of the deformation mechanisms [74]. For traditional coarse-grained metals, $\Omega$ is typically on the order of $b^{3}-10 b^{3}$ for surface dislocation nucleation, whereas $\Omega$ is as large as $100 b^{3}-1000 b^{3}$ for the homogeneous (bulk) dislocation nucleation. Here $b$ is the magnitude of the Burgers vector [52]. Since the strain rate sensitivity is inversely proportional to activation volume, i.e., $m \propto \frac{k_{B} T}{\sigma \Omega}$, the strain rate dependence of surface dislocation nucleation (heterogeneous) is larger than that of homogeneous dislocation nucleation in bulk metals. Here $k_{\mathrm{B}} T$ is the thermal energy with $k_{\mathrm{B}}$ being the Boltzmann constant and $T$ the absolute temperature in Kelvin. Fig. 3b shows the $\Omega$ value as a function of stress associating with the homogeneous dislocation nucleation in the present. For $\sigma<4.25 \mathrm{GPa}$, the activation volume decreases with increasing stress slowly, with the $\Omega$ value in range of $80 b^{3}-95 b^{3}$, which is consistent with the recent experimental measurements [75, 76]. While for $4.25 \mathrm{GPa}<\sigma<5.25 \mathrm{GPa}$, the activation volume also increases mildly as stress increases, with the $\Omega$ value in range of $35 b^{3}-55 b^{3}$. This activation volume is much lower than that in low stress region $(\sigma<4.25 \mathrm{GPa})$ and that in the conventional FCC metals, suggesting that the thermally activated process in MEAs is much more sensitive to temperature and strain rate than that in conventional FCC metals [52]. This is confirmed by the peak stresses 

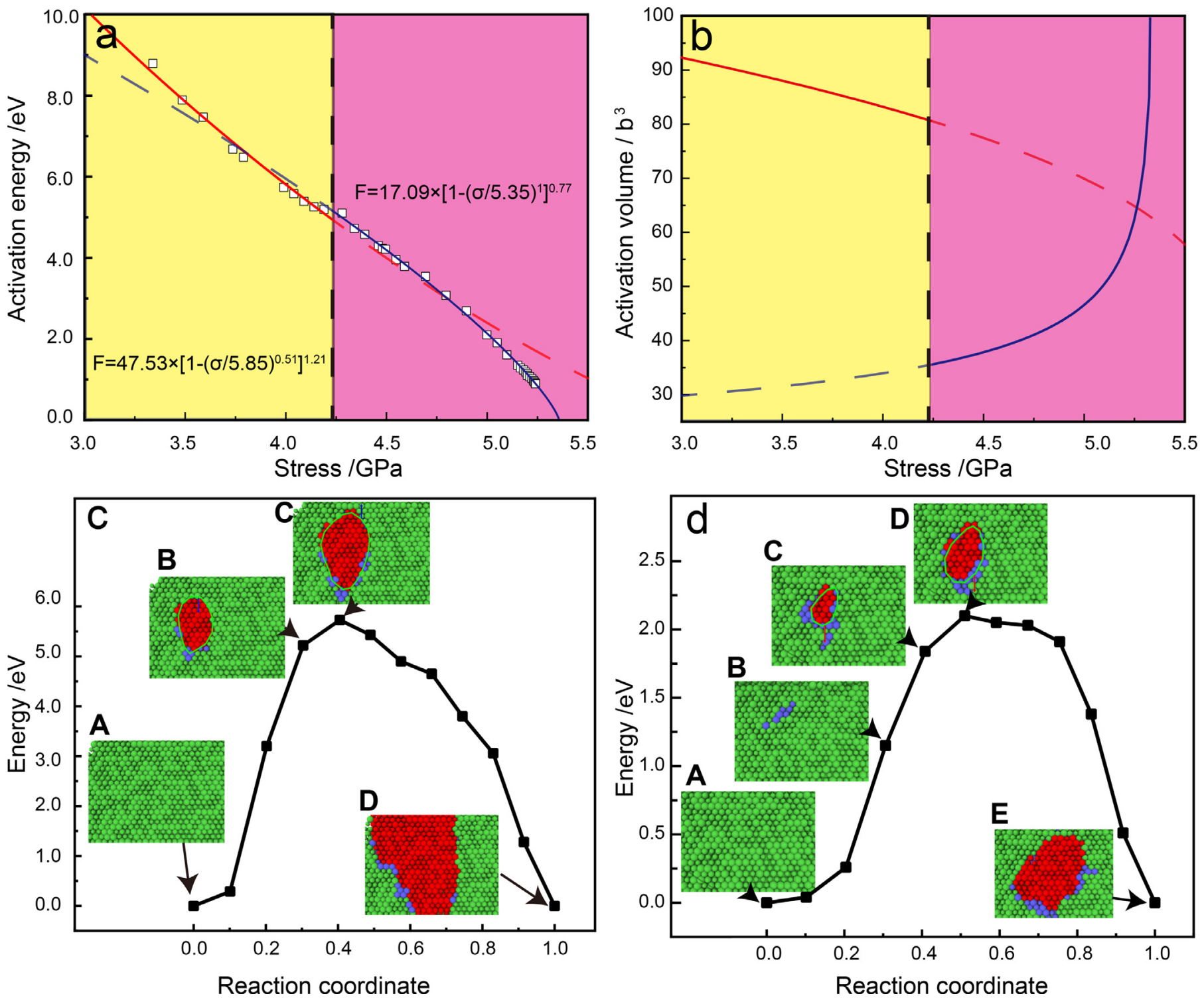

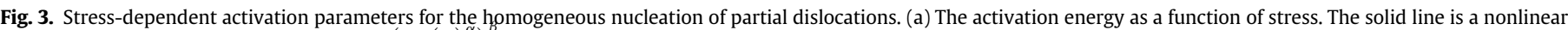

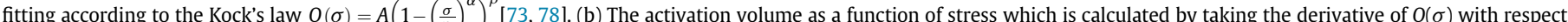

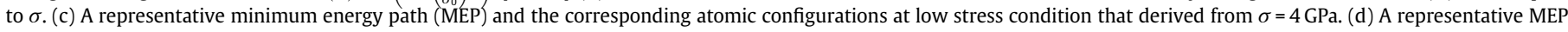
and the corresponding atomic configurations at high stress condition that derived from $\sigma=5 \mathrm{GPa}$.

shown in Fig.S1 in the Supplemental Materials, where they are more strain rate sensitive at high strain rates $\left(5.0 \times 10^{9}-2.5 \times 10^{10} \mathrm{~s}^{-1}\right)$ than that at low strain rates $\left(10^{8}-10^{9} \mathrm{~s}^{-1}\right)$. At very high stress levels near the ideal dislocation-nucleation stress of $\sigma>5.25 \mathrm{GPa}$, the activation volume increases dramatically with stress, suggesting a strong stress dependent nucleation process which is more "athermal" than the rest stress region [54]. The physical scenario of dislocation nucleation here is in strong contrast with the usual observation of one continuous mechanism up to athermal stress [52, 77], which implies strong LCO effect on the nucleation dominated plastic mechanism in chemical complex alloys.

The corresponding atomic configurations of the MEP of dislocation nucleation at relatively low and high stress regimes are displayed in Fig. 3c and d. Here we take the cases of $\sigma=4 \mathrm{GPa}$ and $\sigma=5 \mathrm{GPa}$ as examples. For the high stress region as shown in Fig. $3 \mathrm{~d}$, the MEP can be divided into four successive stages. In the first stage ( $A \rightarrow B$ in Fig. 3d), some perfect fcc atoms rearrange into a bcc structure, with a higher energy state of about $1.15 \mathrm{eV}$ with respect to the initial state. In the second stage $(B \rightarrow C)$, a small dislocation loop enclosing a stacking fault (the red hcp atoms) is nucleated based on the bcc defect clusters. The energy difference between $B$ and $C$ is $0.7 \mathrm{eV}$. This small dislocation loop is energetically less stable and needs further expansion to a bigger and more stable dislocation loop $(C \rightarrow D)$, with an energy difference of $0.3 \mathrm{eV}$. Once a stable dislocation loop is formed it prefers to further expand rather than shrink, being accompanied by a quick decrease in energy $(D \rightarrow E)$. Stress is totally relaxed after reaching to the final state $(E)$ with a stable SF structure. However, as shown in Fig. 3c, in the low stress region, the transition process only can be divided into three stages, i.e., formation $(A \rightarrow B)$, stabilization $(B \rightarrow C)$ and extension of dislocation loop $(C \rightarrow D)$, which misses a clear local fcc-to-bcc transition before dislocation nucleation. It should be pointed out that the bcc cluster shown in the front of the SFs are actually generated after the formation of SF (dislocation loop), which is an atomic mechanism for the propagation of SF (dislocation loop) in this CrCoNi MEA [79].

Note that the activation volume of homogeneous dislocation nucleation in single crystal calculated here is nearly two orders of magnitude greater than those measured by nano-indentation of 

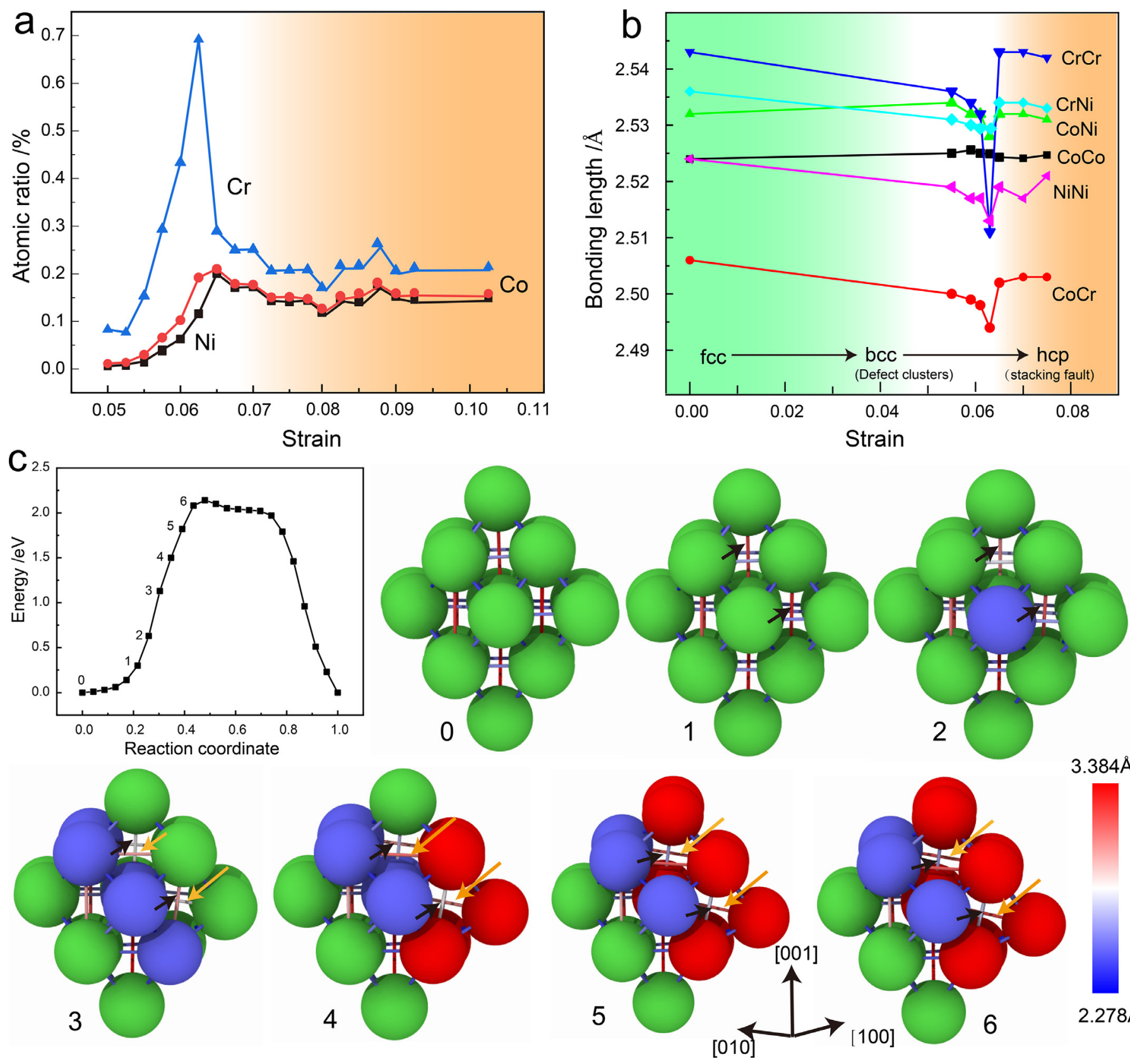

$3.384 \AA$

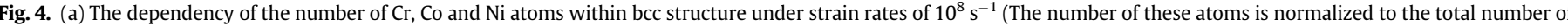

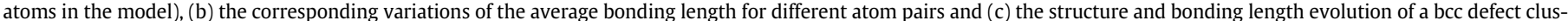

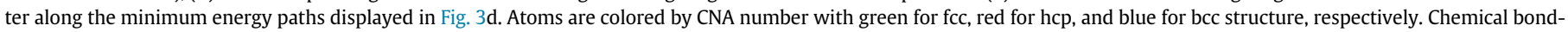
ing are colored by their length as scaled in color bar. (For interpretation of the references to colour in this figure legend, the reader is referred to the web version of this article.)

CrCoNi MEA [80] and HEAs [81] as well as pure metals [82]. The experimental sample usually contain plenty of defects such as vacancy and pre-existing dislocation, which cannot be completely eliminated by annealing [83]. In the presence of pre-existing dislocations, the displacement burst could be associated with the dislocation multiplication rather than nucleation and the dislocation loop expansion could be the dominating mechanism [84]. Besides the dislocation can also nucleate at a pre-existing vacancy or vacancy cluster [53]. Therefore, the pop-in events under nanoindentation are usually heterogeneous dislocation nucleation processes with small activation volume, even sometimes involve diffusive mechanisms. Our simulations are implemented in a defect-free sample, and the dislocation are nucleated homogeneously as shown in Figs. 1-3.

If fed into the rate equation, these large barriers ( $>5 \mathrm{eV}$, Fig. 3a) in low stress region would lead to an unreasonable time scale for dislocation nucleation. However, the energy difference between a fully nucleated dislocation and the bcc defect clusters is $\sim 0.7 \mathrm{eV}$, which will give a reasonable nucleation rate comparable to a typical laboratory experiments (time scale of seconds to hours) at room temperature. Therefore, the nucleation of dislocation loop can only occur at a very high stress condition assisted by the nanoscale bcc-like defect cluster formation. Several simulations have shown that the homogeneous nucleation of dislocation is assisted by the similar defect structures, which was considered to be the result of thermal fluctuations within corresponding atomically small activation volumes $[53,85,86]$. On the other hand, the fact that numerous of new small SFs that enclosed by partial dislocation loops grow in the early stage indicates a high dislocation nucleation rate at high strain rate loading (see Fig.S2 in the Supplemental Materials). This may result from the large numbers of bcc-like atoms ( $~ 7 \%$, see Fig.S2) during the 
onset of dislocation nucleation, which are much higher than that of low rate $(\sim 0.7 \%$, See Fig. 1$)$. All of these imply that the formation of nanoscale defect cluster is vital to the nucleation of dislocation in the generic chemically inhomogeneous alloys.

\subsection{Formation of bcc-like defect cluster}

As shown, the formation of this bcc-like defect cluster can be caused by either thermal fluctuation (Fig. 1) or stress (Fig. 3). To further study the formation mechanism of these bcc-like defect clusters, we examine the atomic structural evolution of the bcc defect clusters during compression at strain rate of $10^{8} \mathrm{~s}^{-1}$ and along the minimum energy paths at high stress condition of $\sigma=5 \mathrm{GPa}$. The results are shown in Fig. 4, in which Fig. 4a show the evolution of the numbers of $\mathrm{Cr}, \mathrm{Co}$ and $\mathrm{Ni}$ atoms with bcc-like environment. In the conventional metals, the related structural rearrangements during deformation do not occur for isolated atoms and the defect nucleation sites are usually determined by thermal fluctuations [87]. But in the case of CrCoNi MEA studied here, the atom fraction of $\mathrm{Cr}$ in these bcc defect clusters is much higher than others, implying a different mechanism for the defect formation during deformation.

This chemical inhomogeneity of the defect structure is related to the property of the chemical bonding of different atom pairs [88]. Fig. $4 \mathrm{~b}$ show the change of the bonding length of different atom pairs as strain increases. In the elastic stage, all the bonding lengths decrease with different rates except the $\mathrm{Co}-\mathrm{Ni}$ bonding, which suggests obvious differences in the nature of different chemical bonds. All the bonding lengths including $\mathrm{Co}-\mathrm{Ni}$ pair are reduced rapidly when the bcc defect clusters begin to form at strain of $\sim 5 \%$. The minimum of the bonding lengths is observed at compressive strain of $\sim 6.3 \%$, corresponding to the maximum number of bcc defect atoms (as shown in Fig. 4a). The bcc atoms decrease but the bonding lengths increase as the deformation proceeds to the dislocation dominated stable state. The dramatic reduction of the bond lengths during the formation of the bcc defect clusters implies a lattice collapse mechanism for such cluster formation, which is similar to the well accepted Bain model for the fcc-to-bcc phase transition [89]. The drastic contraction of $\mathrm{Cr}-\mathrm{Cr}$ bonding during the defect formation suggests that the $\mathrm{Cr}-\mathrm{Cr}$ bonding is weaker. The defect cluster prefers to originate from the collapse of the $\mathrm{Cr}-\mathrm{Cr}$ bonding, conforming to the high atom ratio of $\mathrm{Cr}$ in the bcc defect clusters as demonstrated in Fig. 4a. This evolution mechanism of the bcc defect clusters is identical for high rate loading (see Fig.S3). Fig. 4c details the evolution of the atomic structure and bonding length of a small bcc defect cluster along the minimum energy paths at high stress condition of $\sigma=5 \mathrm{GPa}$. It reveals an obvious structure collapse process during bcc formation characterized by an evident shorten of bonding along [001] direction (directed by black arrows), especially during the formation of a double-layered BCC cluster (denoted as No. 3 configuration in Fig. 4c). While the formation of hcp atoms are also accompanied by a stretch of bonding (directed by yellow arrows), corresponding to the recovery of bonding length at strain $>\sim 6.3 \%$ shown in Fig. $4 \mathrm{~b}$. This evolution process coincides well with the MD simulations shown in Fig. $4 \mathrm{~b}$, which obeys the well-accepted Bain model of FCC $\rightarrow$ BCC phase transition.

\subsection{Electronic structure mechanism}

But there are still two open questions remaining. 1) Is the defectmediated dislocation nucleation mechanism relevant to the accuracy of the empirical interatomic potential used in the MD simulations? And 2) what is the governing mechanism of the lattice and bond collapse during the formation of the bcc-like defect clusters? To answer these questions, first-principles calculations are performed here to further verify the proposed nucleation mechanism in atomistic simulations, and reveal a possible electronic structure mechanism.
Fig. 5 shows the stress-strain curve and the corresponding structural evolution of a RSS-CrCoNi MEA supercell of 228 atoms during the first-principles compression along [001] crystallographic orientation. Overall, the stress-strain behavior obtained by DFT agrees well with MD simulations. Stress increases monotonously in the elastic region and then declines sharply due to the formation of dislocation (or SF) that indicates the initiation of plastic deformation. As expected, there also appears a local bcc defect cluster before the formation of SF, in analogy with the results predicted by MD simulations. Closer comparison of Fig. 5 with Fig. 1 would reveal that the local bcc defect appears at the yielding point of the DFT calculations, whereas it arises before yielding for the MD simulation. This difference can be attributed to the atomic thermal vibration in MD, while the DFT is athermal compression at $0 \mathrm{~K}$. For the MD simulations performed at $300 \mathrm{~K}$, the atoms fluctuate near their equilibrium positions, which is easy to deviate strongly from the equilibrium position and sometimes leads to the formation of defect clusters under combined conditions of stress and temperature. In this case, the defect clusters are unstable and may vanish during relaxation [87]. At high strain rate loading, the structural relaxation of the bcc defect atoms are suppressed (no enough relaxation time allowed) and thus the survived defect atoms are more abundant than that at low strain rate (see Fig. S2). While for the first-principle calculations, the atoms are all in their ground state such that no thermal vibration involved, it is reasonable that the bcc defects are only induced by large stress which promotes atomic rearrangement near and after yielding. Below the critical stress in MD, the formation of the bcc defects is attributed to thermal vibration, which is energetically unfavorable and thus unstable. However, in the case of DFT, the defect clusters can be activated if only the stress reach the critical value in stress-strain curve. In this case, the bcc defect cluster can be directly formed inherited from the perfect fcc lattice, which is consistent with the MEP of dislocation nucleation at high stress $\sigma=5 \mathrm{GPa}$, calculated by FENEB (Fig. 3d).

We also notice that the bcc defect atoms are also of high concentration of $\mathrm{Cr}$ element in the current DFT calculations, which again reproduces the scenario predicted by MD simulations. As shown in Fig. 4, there is consistent decrease in bond length with increasing strain during multiplication of the bcc clusters. This suggests a stressassisted formation mechanism of the defect clusters. To obtain further understanding of the electronic origin of the bcc-like defect generation, the electron localization function (ELF) at different strains of $\varepsilon=0, \varepsilon=0.085, \varepsilon=0.092$ and $\varepsilon=0.099$ are plotted in Fig. 6 . The ELF are given by $\quad \mathrm{ELF}=\frac{1}{1+\left(\frac{D}{D_{h}}\right)^{2}}, \quad$ where $\quad D=\frac{1}{2} \sum_{i}\left|\nabla \varphi_{i}\right|^{2}-\frac{1}{8} \frac{|\nabla \rho|^{2}}{\rho} \quad$ and $D_{h}=\frac{3}{10}\left(3 \pi^{2}\right)^{5 / 3} \rho^{5 / 3}, \varphi_{i}$ is the wave function built from Hartree-Fock or Kohn-Sham orbitals [90]. The ELF values are range from 0 to 1 , where 1 corresponds to perfect localization. The strains are corresponding to the four typical structures as shown in the insets of Fig. 5. Briefly, the higher the ELF value between atoms, the more localized the electronic behaviors, and the more dominant the covalent bonding nature. Fig. 6a reveals a concentrated ELF distribution around $\mathrm{Cr}$ atoms, implying a localized electronic behavior of $\mathrm{Cr}$ atoms and a covalent bonding character of $\mathrm{Cr}-\mathrm{Cr}$ bonding. This is in strong contrast with the nonlocal nature of ELF function around either $\mathrm{Co}$ or $\mathrm{Ni}$ atoms. This covalent character makes the $\mathrm{Cr}-\mathrm{Cr}$ bonding more directional, which would exhibit a poor deformation compatibility. This will induce possible stress concentration on $\mathrm{Cr}$ atoms during loading. Further, this directional $\mathrm{Cr}-\mathrm{Cr}$ bonding would collapse under very high stress, leading to the formation of bcc defect clusters, as demonstrated in Fig. 4. Note that the ELF is slightly decreased when the bcc defect atoms are formed (compare Fig. $6 b, c$, to a). However, these variations are too small to produce an essential change of the $\mathrm{Cr}-\mathrm{Cr}$ covalent bonding character. Further deformation results in no pronounced change in the ELF distribution, but the new bcc atoms (and hcp atoms) still 


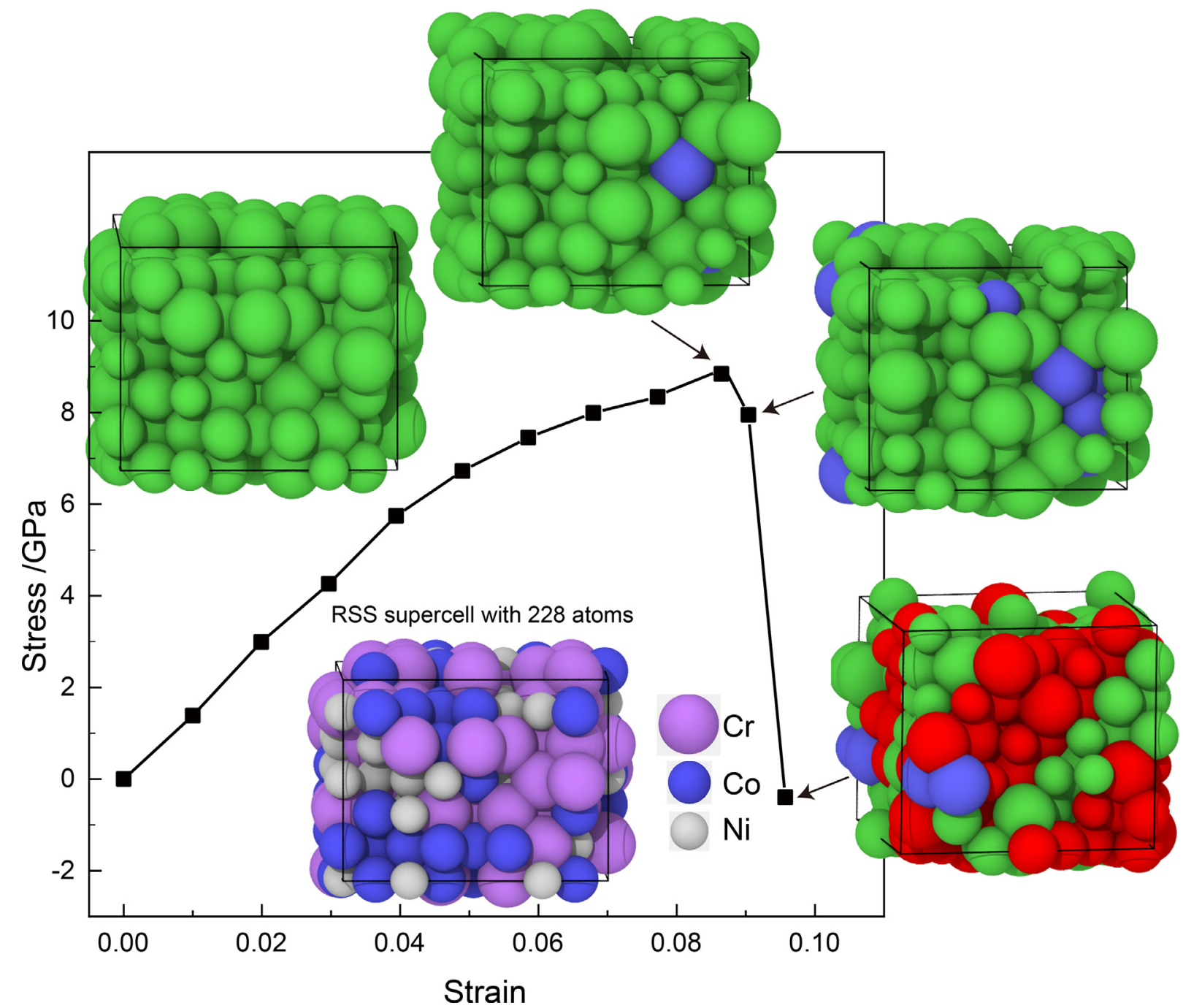

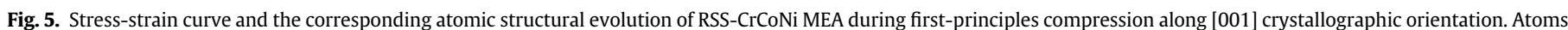

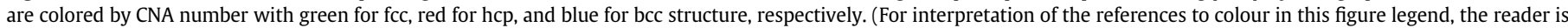
referred to the web version of this article.)

appear around the concentrated ELF region with uninterrupted $\mathrm{Cr}$ atoms (as shown in Fig. 6b-d). In a word, the localized electronic distribution of the $\mathrm{Cr}$ atoms that possess covalent bonding nature can be severed as the soft region, in which stress concentration take place, and consequently the bcc-like defects emerge after bonding collapses during deformation. (a) $\quad \varepsilon=0$

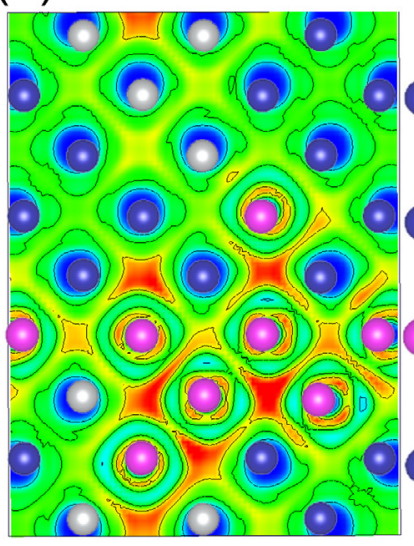

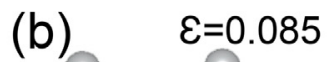

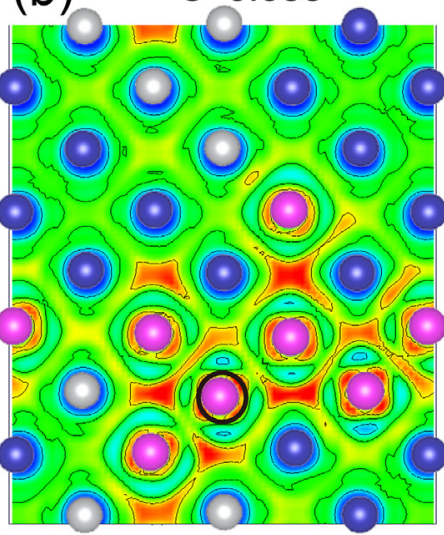

(c) $\varepsilon=0.092$

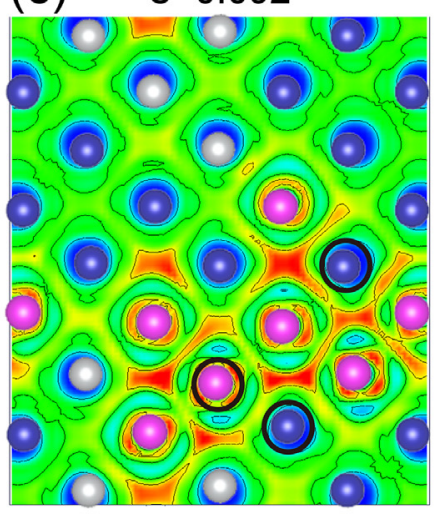

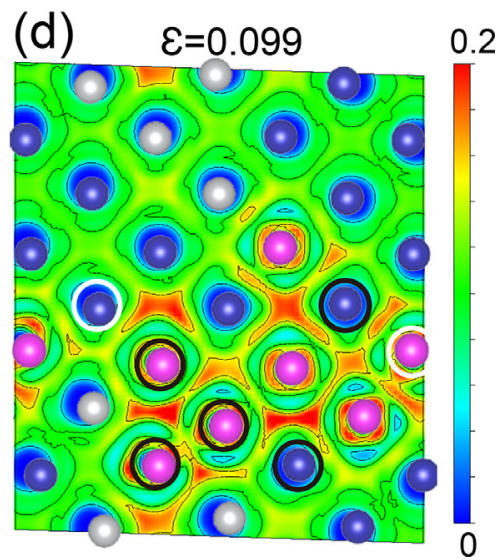

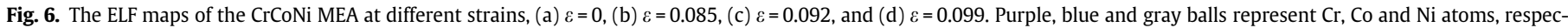

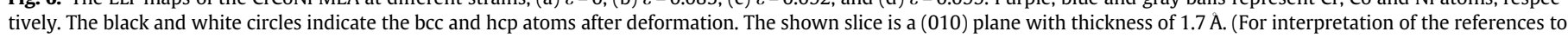
colour in this figure legend, the reader is referred to the web version of this article.) 
To further explore the electronic level bonding properties among the $\mathrm{Cr}-\mathrm{Cr}, \mathrm{Cr}-\mathrm{Co}, \mathrm{Cr}-\mathrm{Ni}, \mathrm{Co}-\mathrm{Co}, \mathrm{Co}-\mathrm{Ni}$ and $\mathrm{Ni}-\mathrm{Ni}$ pairs in the local region with a concentrated ELF distribution (Fig. 6a), the total electron charge density and the local density of states (LDOS) are calculated for the undeformed CrCoNi MEA. Through the charge density shown in Fig. 7a, one can see that the chemical bonding is already anisotropic and asymmetric in the undeformed configuration, caused by the heterogeneous local chemical environment. Fig. 7b show the LDOS for the atoms in different sites that are also plotted in the light of the nearest atom pairs $(\mathrm{Cr} 1-\mathrm{Cr} 2, \mathrm{Cr} 1-\mathrm{Co} 1, \mathrm{Cr} 1-\mathrm{Ni1}, \mathrm{Co} 2-\mathrm{Co} 3, \mathrm{Co} 3-$ $\mathrm{Ni} 2$ and $\mathrm{Ni2}-\mathrm{Ni} 3$ ). As shown, the spin-up DOS of $\mathrm{Cr} 1$ atom is splitted into two peaks: one is located in the right of Fermi level $\left(E_{\mathrm{Fermi}}\right)$, and the other is located very near to $E_{\mathrm{Fermi}}$. Different from $\mathrm{Cr} 1$, the spinup DOS of $\mathrm{Cr} 2$ atom shows only one peak in the right of $E_{\mathrm{Fermi}}$. The difference in LDOS between adjacent $\mathrm{Cr}$ atoms could be attributed to the dissimilarity in the local environments. For example, the $\mathrm{Cr} 2$ atom is also a nearest neighbor of Ni1 atom, so that DOS of $\mathrm{Cr} 2$ is much affected by the extended electronic states of Ni1, as shown in the Cr2-Ni1 DOSs in Fig. 7b (left middle panel). Moreover, the distinct DOS peaks of $\mathrm{Cr} 1$ atom indicate a localized electronic behavior, which tends to form covalent bonding with other atoms. The charge density (Fig. 7a) between $\mathrm{Cr} 1$ and $\mathrm{Cr} 2$ are much lower than that of $\mathrm{Cr} 1-\mathrm{Co} 1$

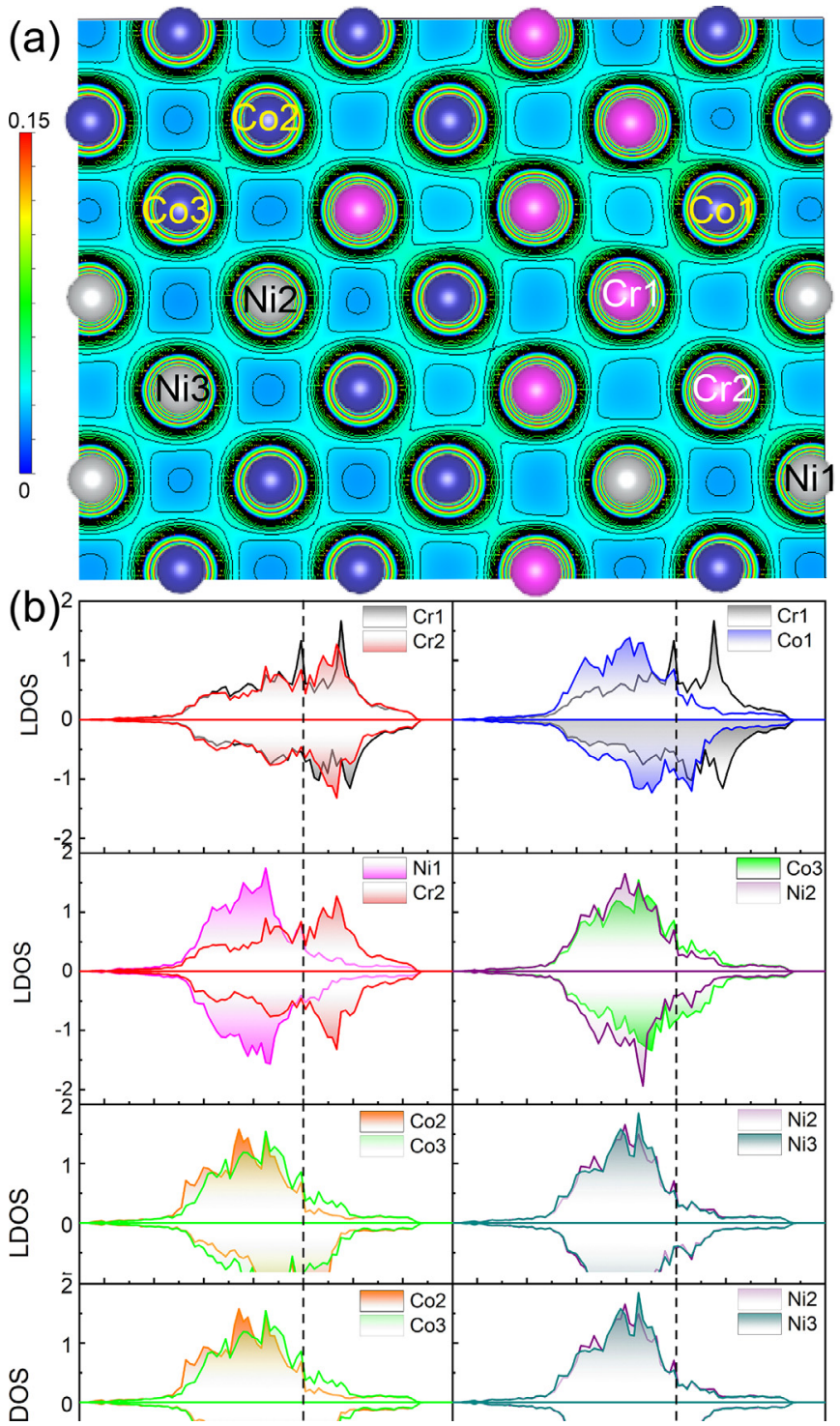

Fig. 7. (a) Total charge densities on a (010) plane, and (b) the LDOS for the selected nearest neighbor atoms in the undeformed CrCoNi MEA. and $\mathrm{Cr} 2-\mathrm{Ni} 1$, suggesting that the bonding of $\mathrm{Cr} 1-\mathrm{Cr} 2$ are much weaker than that of the $\mathrm{Cr} 1-\mathrm{Co} 1$ and $\mathrm{Cr} 2-\mathrm{Ni} 1$ bonding. Note that the shapes of the DOS curves are highly overlap for the $\mathrm{Co}-\mathrm{Co}, \mathrm{Co}-\mathrm{Ni}$ and $\mathrm{Ni}-\mathrm{Ni}$ bonding, all of which indicate metallic bonding nature. The metallic nature of the bonding is usually associated with coordinated deformation behavior. Whereas the $\mathrm{Cr}$-atom-rich region with directional covalent bonding nature would cause nonuniform deformation and thus stress concentration appears. Thereafter, the weak $\mathrm{Cr}-\mathrm{Cr}$ bonding would be compressed severely which finally leads to the formation of bcc-like defects. Finally, it is interesting to note that the spin-up and spin-down DOSs of $\mathrm{Cr}$ and Co atoms vary significantly based on different positions. It may indicate that the magnetism effect due to different local chemical environment also play a significant role in the deformation of such chemically complex alloys [91].

In summary, the localized, directional bonding between $\mathrm{Cr}$ atoms imply a poor deformation compatibility, which may induce possible stress concentration during loading. While the weak bonding between $\mathrm{Cr}$ atoms inferred by the low charge density suggest a relatively low load-bearing capacity that promotes local structure collapse under stress. After which, formation of bcc defect cluster is possible, which serves as a precusor for partial dislocation nucleation. Actually, the localized electronic behavior of $\mathrm{Cr}$ atom is partly responsible for the weak bonding between $\mathrm{Cr}$ atoms. The localized electronic behavior, directional bonding and weak bonding of $\mathrm{Cr}$ atoms jointly promote the local structural transformation of $\mathrm{Cr}$ atoms and the following associated dislocation nucleation.

\subsection{Role of local chemical order}

Recall that some specific chemical bonds are preferred than others and affect the deformation behaviors in the generic chemically complex alloys, etc., MEAs and HEAs. Our MD and DFT results strongly suggest that the weak $\mathrm{Cr}-\mathrm{Cr}$ bonding originates from its localized electronic behaviors. The electronic localization also motivates the formation of bcc-like defect cluster, and thus facilitates the nucleation of dislocation (or SF) in $\mathrm{CrCoNi}$. Therefore one may expect that the LCO associated with $\mathrm{Cr}-\mathrm{Cr}$ pairs would affect the mechanical behavior of the CrCoNi MEA. In order to test the assumption, we perform extra MD compression tests on CrCoNi MEAs with different level of LCO, but the same chemical ratio 1:1:1. In consideration of the fact that stress drops more slowly with a finite slope at relatively higher strain rates after yielding, a higher strain rate of $10^{10} / \mathrm{s}$ are used in the extra MD compression tests to the stress-strain behaviors during incipient plasticity stage of the samples with different local chemical order. There is a positive strain rate sensitivity, which leads to increased nucleation stress for dislocations. In this circumstance, the dislocation nucleation behaviors are more localized, which yields smaller value of activation volume as rationalized by the stressdependent activation enthalpy shown in Fig. 3. Therefore, direction MD simulations and NEB calculations of dislocation nucleation render the same scenario on the stress dependence, which is critical for understanding the low strain rate conditions used in a real mechanical experiment.

Fig. 8 shows the stress-strain curves of these MEAs. Overall, all the samples possess a similar stress-strain behavior as described for RSS samples in Fig. 1 and Fig. S1. Nevertheless, there are many specific differences among these samples. For example, the low $\mathrm{Cr}$ sample $(22 \% \mathrm{Cr})$ exhibits the highest elastic modulus (the slope of the linear elastic region) of $133 \mathrm{GPa}$, which is $\sim 3$ times of the high Cr sample ( $44 \% \mathrm{Cr}, 44 \mathrm{GPa}$ ), and $\sim 1.5$ times of RSS sample (medium $\mathrm{Cr}$ of $33.33 \%$, $90 \mathrm{GPa}$ ). Similarly, the yield strength shows the same trend with elastic modulus in an order of low $\mathrm{Cr}>\mathrm{RSS}$ (medium $\mathrm{Cr}$ ) $>$ high $\mathrm{Cr}$. The trend in modulus and strength supports the conclusion of relatively weak $\mathrm{Cr}-\mathrm{Cr}$ bond as demonstrated in the electronic structure analysis, as shown in Figs. 6 and 7. It also suggests that the $\mathrm{Cr}$ content plays a crucial role in the mechanical property, just as it does to the physical 

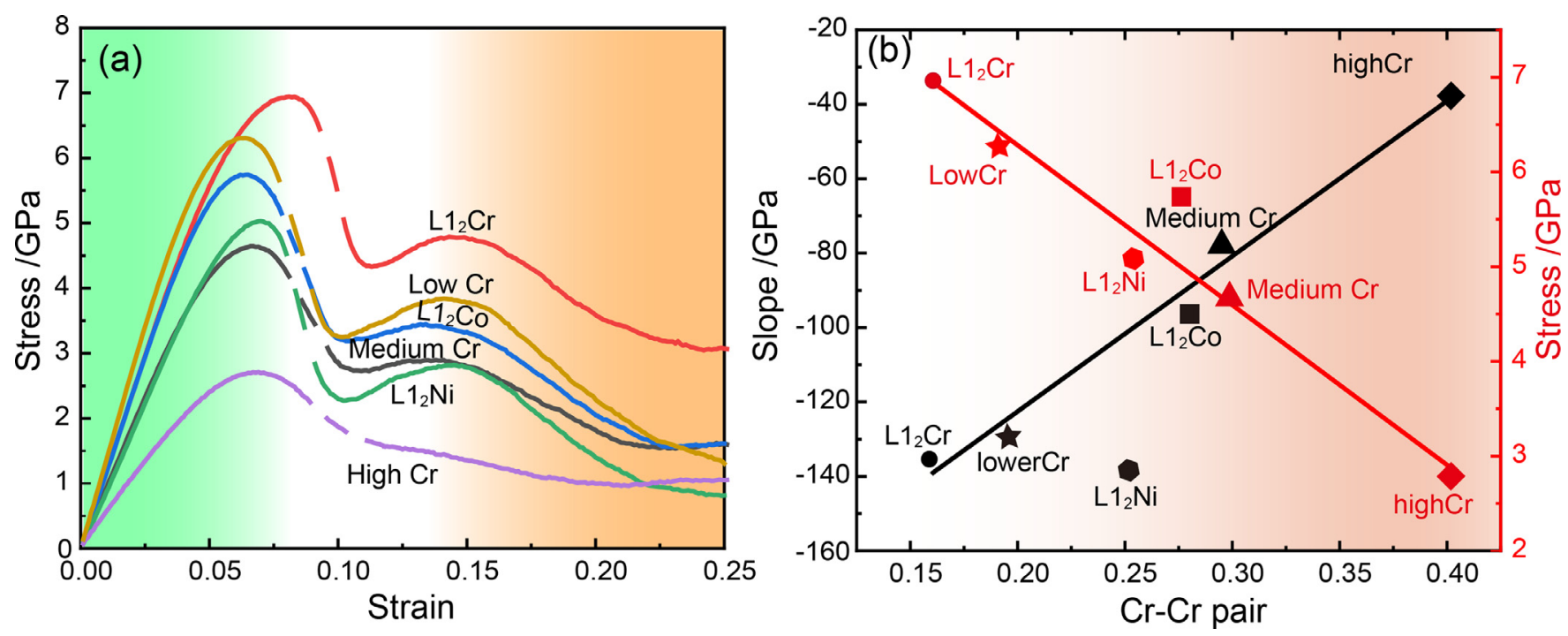

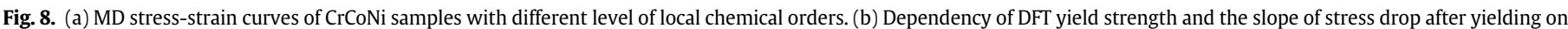
the ratio of $\mathrm{Cr}-\mathrm{Cr}$ pairs. The solid lines are served as guide for the eyes.

properties [92]. Similar trend also reported in FeNiCr MEA, in which shear strength at $0 \mathrm{~K}$ is decrease with increasing $\mathrm{Cr}$ content [36]. In addition to $\mathrm{Cr}$ content, the mechanical properties are also significantly altered by the pair distribution i.e., LCO of the samples. The presence of the preferential chemical order results in an increase of the yield strength when moving from the absolute RSS to the alloyed $\mathrm{L} 1_{2}$ structures, as shown in Fig. 8a. The result is consistent well with the recent experimental observation which reveals that the LCO and thus the strength can be tailored through aging [42]. Careful observations also reveal that the stress relaxation (stress drop) average slope between the upper yield point and the lower yield point (the dashed regions of stress-strain curves shown in Fig. 8a), which represents the roughness of MEP for dislocation nucleation and thus incipient plasticity, varies strongly with the $\mathrm{Cr}$ content and the LCO.

The essential distinction between the samples with higher degree of LCO and RSS configurations is the distribution of the chemical bondings, i.e., random distribution or biased bonding. Therefore, the effect of LCO on the properties is largely dependent on the nature and spatial distribution of some special chemical pairs. For example, Li et al. found that the SFE is more closely related to the degree of $\mathrm{Co}-\mathrm{Cr}$ chemical order and suggested a stronger effect of dislocation pinning in the Co$\mathrm{Cr}$ rich region [44]. Similarly, our results show a remarkable linear correlation between the proportion of $\mathrm{Cr}-\mathrm{Cr}$ pair in the alloy and the stress drop slope of the stress-strain curve as well as the yield strength, as plotted in Fig. 8b. The yield strength is inversely proportional to the number of $\mathrm{Cr}-\mathrm{Cr}$ pair. In general, the strength of a material is determined by the weak part rather than the stronger one. The increase of weak $\mathrm{Cr}$ - $\mathrm{Cr}$ pairs leads to increased soft regions, which are apt to experience nonuniform deformation and even failure. Fig. 8b also shows a proportional relationship between the stress drop slope and the proportion of $\mathrm{Cr}-\mathrm{Cr}$ pairs. This result is consistent well with the assumption that the nucleation of dislocation, i.e., incipient plasticity of single crystalline $\mathrm{CrCoNi}$ alloy, is assisted by the nanoscale bcc-like defect cluster, the formation of which is associated with collapse of $\mathrm{Cr}-\mathrm{Cr}$ bonding.

\section{Conclusions}

We have studied the incipient plasticity behaviors in a chemically complex CrCoNi MEA by the combined MD simulations and DFT calculations. The MEP calculated by FENEB reveals a nanoscale bcc-like defect cluster mediated dislocation nucleation mechanism, which give a reasonable nucleation rate comparable to a typical laboratory experiments (time scale of seconds to hours) at room temperature.
This nucleation mechanism is confirmed by DFT compression test on a RSS CrCoNi alloy based on a SQS supercell. The small activation volumes for dislocation nucleation imply enhanced strain rate and temperature sensitivity in contrast with dislocation nucleation in conventional metals, which is also in agreement with recent experimental observations.

The local bcc-like defect cluster is preferentially generated on $\mathrm{Cr}$ atoms, accompanied by dramatic contraction of $\mathrm{Cr}-\mathrm{Cr}$ bonding. DFT calculations reveal a localized electronic behavior of $\mathrm{Cr}$ atoms and a weak $\mathrm{Cr}-\mathrm{Cr}$ bonding, which facilitates the formation of nanoscale bcc-like defects served as a precursor for the homogeneous dislocation nucleation. Further MD compressive tests on several specifically designed $\mathrm{CrCoNi}$ alloys with different level of LCO reveal interesting correlation between mechanical property and LCO. The yield strength is found to be inversely proportional to the fraction of $\mathrm{Cr}-\mathrm{Cr}$ pairs. However, the slope of stress drop after yielding, which may reflect the roughness of dislocation nucleation pathway, increases with increasing $\mathrm{Cr}-\mathrm{Cr}$ pairs. The atomistic and electronic level insights obtained here is informative to a concrete understanding of the unique dislocation nucleation mechanism and incipient plasticity in the generic chemically complex alloys. The proposed correlation between mechanical property and LCO should benefit material designs in the recently emerging and promising high-entropy and medium-entropy alloys.

\section{Declaration of Competing Interest}

We confirm that there is no conflict of interest that could have appeared to affect the work reported in this paper.

\section{Acknowledgment}

Thanks to Qingjie Li (Massachusetts Institute of Technology) for discussions on FENEB. This work is supported by the NSFC Basic Science Center Program for "Multiscale Problems in Nonlinear Mechanics" (No.11988102), the NSFC (No. 11790292 and No. 11672299), the National Key Research and Development Program of China (No. 2017 YFB0702003 and No. 2017YFB0701502), the Strategic Priority Research Program (No. XDB22040302 and No. XDB22040303), the Key Research Program of Frontier Sciences (Grant No. QYZDJSSWJSC011), Science Challenge Project (No. TZ2016001), the Youth Promotion Association of Chinese Academy of Sciences (No. 2017025), 
and the opening project of State Key Laboratory of Explosion Science and Technology (No. KFJJ18-14 M).

\section{Supplementary materials}

Supplementary material associated with this article can be found in the online version at doi:10.1016/j.actamat.2020.05.042.

\section{References}

[1] B. Cantor, I.T.H. Chang, P. Knight, A.J.B. Vincent, Microstructural development in equiatomic multicomponent alloys, Mater. Sci. Eng. A 375-377 (2004) 213-218.

[2] D.B. Miracle, O.N. Senkov, A critical review of high entropy alloys and related concepts, Acta Mater 122 (2017) 448-511

[3] J.W. Yeh, S.K. Chen, S.J. Lin, J.Y. Gan, T.S. Chin, T.T. Shun, C.H. Tsau, S.Y. Chang, Nanostructured high-entropy alloys with multiple principal elements: novel alloy design concepts and outcomes, Adv. Eng. Mater 6 (2004) 299-303.

[4] Y.F. Ye, Q. Wang, J. Lu, C.T. Liu, Y. Yang, High-entropy alloy: challenges and prospects, Mater. Today 19 (2016) 349-362.

[5] Z.Z. Li, S.T. Zhao, Robert O. Ritchie, Marc A. Meyers, Mechanical properties of highentropy alloys with emphasis on face-centered cubic alloys, Prog. Mater. Sci. 102 (2019) 296-345

[6] Y. Zhang, T.T. Zuo, Z. Tang, Michael C. Gao, Karin A. Dahmen, Peter K. Liaw, Z.P. Lv, Microstructures and properties of high-entropy alloys, Prog. Mater. Sci. 61 (2014) $1-93$.

[7] Evan Ma, Unusual dislocation behavior in high-entropy alloys, Scr. Mater. 181 (2020) 127-133.

[8] E.P. George, W.A. Curtin, C.C. Tasan, High entropy alloys: a focused review of mechanical properties and deformation mechanisms, Acta Mater. 188 (2020) 435-474.

[9] Easo P. George, Dierk Raabe, Robert O. Ritchie, High- entropy alloys, Nature Rev. (2019).

[10] B. Gludovatz, A. Hohenwarter, K.V. Thurston, H. Bei, Z. Wu, E.P. George, R.O. Ritchie, Exceptional damage-tolerance of a medium-entropy alloy $\mathrm{CrCoNi}$ at cryogenic temperatures, Nat. Commun. 7 (2016) 10602.

[11] Z. Zhang, M.M. Mao, J. Wang, B. Gludovatz, Z. Zhang, S.X. Mao, E.P. George, Q. Yu, R.O. Ritchie, Nanoscale origins of the damage tolerance of the high-entropy alloy CrMnFeCoNi, Nat. Commun. 6 (2015) 10143.

[12] S.P. Wang, Evan Ma, J. Xu, Notch fracture toughness of body-centered-cubic (TiZrNbTa) Mo high-entropy alloys, Intermetallics 103 (2018) 78-87.

[13] Z. Zhang, H. Sheng, Z. Wang, B. Gludovatz, Z. Zhang, E.P. George, Q. Yu, S.X. Mao, R.O. Ritchie, Dislocation mechanisms and 3D twin architectures generate exceptional strength-ductility-toughness combination in CrCoNi medium-entropy alloy, Nat. Commun. 8 (2017) 14390

[14] B. Gludovatz, A. Hohenwarter, D. Catoor, E.H. Chang, Easo P George, Robert O Ritchie, A fracture-resistant high-entropy alloy for cryogenic applications, Science 345 (2014) 1153-1158.

[15] Z. Li, K.G. Pradeep, Y. Deng, D. Raabe, C.C. Tasan, Metastable high-entropy dualphase alloys overcome the strength-ductility trade-off, Nature 534 (2016) 227 230.

[16] J.P. Liu, J.X. Chen, T.W. Liu, C. Li, Y. Chen, L.H. Dai, Superior strength-ductility CoCrNi medium-entropy alloy wire, Scr. Mater. 181 (2020) 19-24.

[17] Z.F. Lei, X.G. Liu, Y. Wu, H. Wang, S.H. Jiang, S.D. Wang, X.D. Hui, Y.D. Wu, B. Gault P. Kontis, Dierk Raabe, L. Gu, Q.H. Zhang, H.W. Chen, H.T. Wang, J.B. Liu, K. An, Q.S. Zeng, T.G. Nieh, Z.P. Lu, Enhanced strength and ductility in a high-entropy alloy via ordered oxygen complexes, Nature 563 (2018) 546-550.

[18] T. Yang, Y.L. Zhao, Y. Tong, Z.B. Jiao, J. Wei, J.X. Cai, X.D. Han, D. Chen, A. Hu, J.J. Kai, K. Lu, C.T. Liu, Multicomponent intermetallic nanoparticles and superb mechanical behaviors of complex alloys, Science 362 (2018) 933-937.

[19] Z.Q. Fu, L. Jiang, J.L. Wardini, B.E. MacDonald, H.M. Wen, W. Xiong, D.L. Zhang, Y.Z. Zhou, T.J. Rupert, W.P. Chen, J.L. Enrique, A high-entropy alloy with hierarchical nanoprecipitates and ultrahigh strength, Sci. Adv, 4 (2018) eaat8712.

[20] Y.L. Zhao, T. Yang, Y. Tong, J. Wang, J.H. Luan, Z.B. Jiao, D. Chen, Y. Yang, A. Hu, C.T. Liu, J.J. Kai, Heterogeneous precipitation behavior and stacking-fault-mediated deformation in a CoCrNi-based medium-entropy alloy, Acta Mater. 138 (2017) 72-82

[21] J.Y. He, H. Wang, H.L. Huang, X.D. Xu, M.W. Chen, Y. Wu, X.J. Liu, T.G. Nieh, K. An, Z.P. Lu, A precipitation-hardened high-entropy alloy with outstanding tensile properties, Acta Mater. 102 (2016) 187-196.

[22] W.J. Lu, C.H. Liebscher, F.K. Yan, X.F. Fang, L.L. Li, J.J. Li, W.Q. Guo, G. Dehm, Dierk Raabe, Z.M. Li, Interfacial nanophases stabilize nanotwins in high-entropy alloys, Acta Mater. 185 (2020) 218-232.

[23] P.J. Shi, W.L. Ren, T.X. Zheng, Z.M. Ren, X.L. Hou, J.C. Peng, P.F. Hu, Y.F. Gao, Y.B. Zhong, Peter K Liaw, Enhanced strength-ductility synergy in ultrafinegrained eutectic high-entropy alloys by inheriting microstructural lamellae, Nat. Commun. 10 (2019) 1-8.

[24] M.N. Hasan, Y.F. Liu, X.H. An, J. Gu, M. Song, Y. Cao, Y.S. Li, Y.T. Zhu, X.Z. Liao, Simultaneously enhancing strength and ductility of a high-entropy alloy via gradient hierarchical microstructures, Int. J. Plast. 123 (2019) 178-195.

[25] X.F. Liu, Z.L. Tian, X.F. Zhang, H.H. Chen, T.W. Liu, Y. Chen, Y.J. Wang, L.H. Dai, “Selfsharpening" tungsten high-entropy alloy, Acta Mater. 186 (2020) 257-266

[26] Z. Pu, Y. Chen, L.H. Dai, Strong resistance to hydrogen embrittlement of highentropy alloy, Mater. Sci. Eng. A 736 (2018) 156-166.
[27] Y.K. Zhao, D.H. Lee, M.Y. Seok, J.A. Lee, M.P. Phaniraj, J.Y. Suh, H.Y. Ha, J.Y. Kim, U. Ramamurty, J. Jang, Resistance of $\mathrm{CoCrFeMnNi} \mathrm{high-entropy} \mathrm{alloy} \mathrm{to} \mathrm{gaseous}$ hydrogen embrittlement, Scr. Mater. 135 (2017) 54-58.

[28] H. Luo, W.J. Lu, X.F. Fang, D. Ponge, Z.M. Li, Dierk Raabe, Beating hydrogen with its own weapon: nano-twin gradients enhance embrittlement resistance of a highentropy alloy, Mater. Today 21 (2018) 1003-1009.

[29] K. Ichii, M. Koyama, C.C. Tasan, K. Tsuzaki, Comparative study of hydrogen embrittlement in stable and metastable high-entropy alloys, Scr. Mater. 150 (2018) 74-77.

[30] Z.J. Jiang, J.Y. He, H.Y. Wang, H.S. Zhang, Z.P. Lu, L.H. Dai, Shock compression response of high entropy alloys, Mater. Res. Letters 4 (2016) 226-232.

[31] Z.Z. Li, S.T. Zhao, S.M. Alotaibi, Y. Liu, B.F. Wang, Marc A. Meyers, Adiabatic shear localization in the CrMnFeCoNi high-entropy alloy, Acta Mater. 151 (2018) 424-431.

[32] T.W. Zhang, S.G. Ma, D. Zhao, Y.C. Wu, Y. Zhang, Z.H. Wang, J.W. Qiao, Simultaneous enhancement of strength and ductility in a NiCoCrFe high-entropy alloy upon dynamic tension: micromechanism and constitutive modeling, Int. J. Plast. 124 (2020) 226-246.

[33] T. Egami, M. Ojha, O. Khorgolkhuu, D.M. Nicholson, G.M. Stocks, Local electronic effects and irradiation resistance in high-entropy alloys, Jom 67 (2015) 23452349.

[34] F. Granberg, K. Nordlund, M.W. Ullah, K. Jin, C.Y. Lu, H.B. Bei, L.M. Wang, F. Djurabekova, W.J. Weber, Y. Zhang, Mechanism of radiation damage reduction in equiatomic multicomponent single phase alloys, Phys. Rev. Lett. 116 (2016) 135504.

[35] C.Y. Lu, L.L. Niu, N.J. Chen, K. Jin, T.N. Yang, P.Y. Xiu, Y.W. Zhang, F. Gao, H.B. Bei, S. Shi, M.R. He, I.M. Robertson, W.J. Weber, L.M. Wang, Enhancing radiation tolerance by controlling defect mobility and migration pathways in multicomponent single-phase alloys, Nat. Commun. 7 (2016) 13564.

[36] C. Varvenne, A. Luque, William A. Curtin, Theory of strengthening in fcc high entropy alloys, Acta Mater. 118 (2016) 164-176.

[37] Z.H. Aitken, V. Sorkin, Y.W. Zhang, Atomistic modeling of nanoscale plasticity in high-entropy alloys, J. Mater. Res. 34 (2019) 1509-1532.

[38] Y. Tong, S.J. Zhao, H.B. Bei, T. Egami, Y.W. Zhang, F.X. Zhang, Severe local lattice distortion in $\mathrm{Zr}$ - and/or Hf-containing refractory multi-principal element alloys, Acta Mater. 183 (2020) 172-181.

[39] Q.Q. Ding, Y. Zhang, X. Chen, X.Q. Fu, D.K. Chen, S.J. Chen, L. Gu, F. Wei, H.B. Bei, Y.F. Gao, M.W. Wen, Z. Zhang, T. Zhu, R.O. Ritchie, Q. Yu, Tuning element distribution, structure and properties by composition in high-entropy alloys, Nature 574 (2019) 223-227.

[40] S.J. Chen, H.S. Oh, B. Gludovatz, S.J. Kim, E.S. Park, Z. Zhang, Robert O Ritchie, Q. Yu, Real-time observations of TRIP-induced ultrahigh strain hardening in a dualphase CrMnFeCoNi high-entropy alloy, Nat. Commun. 11 (2020) 1-8.

[41] F.X. Zhang, S. Zhao, K. Jin, H. Xue, G. Velisa, H. Bei, R. Huang, J.Y.P. Ko, D.C. Pagan, J.C. Neuefeind, W.J. Weber, Y. Zhang, Local structure and short-range order in a NiCoCr Solid Solution Alloy, Phys. Rev. Lett. 118 (2017) 205501.

[42] R.P. Zhang, S.T. Zhao, J. Ding, Y. Chong, T. Jia, C. Ophus, M. Asta, Robert O Ritchie A.M. Minor. Verification of short-range order and its impact on the properties of the CrCoNi medium entropy alloy. arXiv preprint arXiv:1912.05610 2019.

[43] A. Tamm, A. Aabloo, M. Klintenberg, M. Stocks, A. Caro, Atomic-scale properties of Ni-based FCC ternary, and quaternary alloys, Acta Mater. 99 (2015) 307-312.

[44] Q.J. Li, H. Sheng, Evan Ma, Strengthening in multi-principal element alloys with ocal-chemical-order roughened dislocation pathways, Nat. Commun. 10 (2019).

[45] Michael Widom, W.P. Huhn, S. Maiti, W. Steurer, Hybrid Monte Carlo/molecular dynamics simulation of a refractory metal high entropy alloy, Metall. Mater. Trans. A 45 (2013) 196-200.

[46] J. Ding, Q. Yu, M. Asta, R.O. Ritchie, Tunable stacking fault energies by tailoring local chemical order in CrCoNi medium-entropy alloys, Proc. Natl. Acad. Sci. U S A 115 (2018) 8919-8924.

[47] Q.Q. Ding, X.Q. Fu, D.K. Chen, H.B. Bei, B. Gludovatz, J.X. Li, Z. Zhang, Easo P George, Q. Yu, T. Zhu, Real-time nanoscale observation of deformation mechanisms in CrCoNi-based medium-to high-entropy alloys at cryogenic temperatures, Mater. Today 25 (2019) 21-27.

[48] I.J. Beyerlein, X.H. Zhang, A. Misra, Growth twins and deformation twins in metals, Annu. Rev. Mater. Res. 44 (2014) 329-363.

[49] D. Rodney, L. Ventelon, E. Clouet, L. Pizzagalli, F. Willaime, Ab initio modeling of dislocation core properties in metals and semiconductors, Acta Mater. 124 (2017) 633-659.

[50] S. Ryu, K. Kang, W. Cai, Entropic effect on the rate of dislocation nucleation, Proc. Natl. Acad. Sci. U S A 108 (2011) 5174-5178.

[51] D.H. Warner, W.A. Curtin, Origins and implications of temperature-dependent activation energy barriers for dislocation nucleation in face-centered cubic metals, Acta Mater. 57 (2009) 4267-4277

[52] T. Zhu, J. Li, A. Samanta, A. Leach, K. Gall, Temperature and strain-rate dependence of surface dislocation nucleation, Phys. Rev. Lett 100 (2008)

[53] C.A. Schuh, J.K. Mason, A.C. Lund, Quantitative insight into dislocation nucleation from high-temperature nanoindentation experiments, Nat. Mater. 4 (2005) 617621.

[54] J.P. Du, Y.J. Wang, Y.C. Lo, L. Wan, Shigenobu Ogata, Mechanism transition and strong temperature dependence of dislocation nucleation from grain boundaries: an accelerated molecular dynamics study, Phys. Rev. B 94 (2016).

[55] A. Sharma, P. Singh, D.D. Johnson, P.K. Liaw, G. Balasubramanian, Atomistic clustering-ordering and high-strain deformation of an Al0.1CrCoFeNi high-entropy alloy, Sci. Rep. 6 (2016) 31028.

[56] S.H. Oh, M. Legros, D. Kiener, G. Dehm, In situ observation of dislocation nucleation and escape in a submicrometre aluminium single crystal, Nat. Mater. 8 (2009) 95-100. 
[57] F. Maresca, William A. Curtin, Theory of screw dislocation strengthening in random BCC alloys from dilute to "High-Entropy" alloys, Acta Mater. 182 (2020) 144-162.

[58] M.A. Tschopp, D.L. McDowell, Influence of single crystal orientation on homogeneous dislocation nucleation under uniaxial loading, J. Mech. Phys. Solids 56 (2008) 1806-1830.

[59] S.I. Rao, C. Woodward, T.A. Parthasarathy, O. Senkov, Atomistic simulations of dislocation behavior in a model FCC multicomponent concentrated solid solution alloy, Acta Mater. 134 (2017) 188-194.

[60] Steve Plimpton, Fast Parallel Algorithms For Short-Range Molecular Dynamics, Sandia National Labs, Albuquerque, NMUnited States, 1993.

[61] S. Melchionna, G. Ciccotti, B. Lee Holian, Hoover NPT dynamics for systems varying in shape and size, Mol. Phys. 78 (1993) 533-544

[62] P.M. Larsen, S. Schmidt, J. Schiøtz, Robust structural identification via polyhedra] template matching, Modell. Simul. Mater. Sci. Eng. 24 (2016) 055007.

[63] A. Stukowski, Visualization and analysis of atomistic simulation data with OVITO-the Open Visualization Tool, Model. Simulat. Mater. Sci. Eng. 18 (2009) 015012

[64] T. Zhu, J. Li, A. Samanta, H.G. Kim, S. Suresh, Interfacial plasticity governs strain rate sensitivity and ductility in nanostructured metals, Proc. Natl. Acad. Sci. U S A 104 (2007) 3031-3036.

[65] Q.J. Li, B. Xu, S. Hara, J. Li, Evan Ma, Sample-size-dependent surface dislocation nucleation in nanoscale crystals, Acta Mater 145 (2018) 19-29.

[66] G. Kresse, J. Furthmüller, Efficiency of ab-initio total energy calculations for metals and semiconductors using a plane-wave basis set, Comput. Mater. Sci. 6 (1996) 15-50.

[67] A. van de Walle, P. Tiwary, M. de Jong, D.L. Olmsted, M. Asta, A. Dick, D. Shin, Y. Wang, L.Q. Chen, Z.K. Liu, Efficient stochastic generation of special quasirandom structures, Calphad 42 (2013) 13-18.

[68] J.P. Perdew, K. Burke, M. Ernzerhof, Generalized gradient approximation made simple, Phys. Rev. Lett. 77 (1996) 3865.

[69] Peter E Blöchl, Projector augmented-wave method, Phys. Rev. B 50 (1994) 17953.

[70] D. Roundy, C.R. Krenn, Marvin L Cohen, J.W. Morris Jr., Ideal shear strengths of fcc aluminum and copper, Phys. Rev. Lett. 82 (1999) 2713

[71] M.L. Falk, J.S. Langer, Dynamics of viscoplastic deformation in amorphous solids, Phys. Rev. E 57 (1998) 7192

[72] I.A. Bryukhanov, V.L. Kovalev, A.V. Larin, Nucleation of dislocations in aluminum alloys with copper, Phys. Solid State 57 (2015) 1807-1817.

[73] W.F. Kocks, A.S. Argon, M.F. Ashby, Thermodynamics and kinetics of slip, Progr. Mater. Sci. 19 (1975) 1-281.

[74] R.J. Asaro, S. Suresh, Mechanistic models for the activation volume and rate sensitivity in metals with nanocrystalline grains and nano-scale twins, Acta Mater. 53 (2005) 3369-3382.

[75] Z.G. Wu, Y.F. Gao, H.B. Bei, Thermal activation mechanisms and Labusch-type strengthening analysis for a family of high-entropy and equiatomic solid-solution alloys, Acta Mater. 120 (2016) 108-119.
[76] S. Hong, J. Moon, S.K. Hong, H.S. Kim, Thermally activated deformation and the rate controlling mechanism in $\mathrm{CoCrFeMnNi} \mathrm{high} \mathrm{entropy} \mathrm{alloy,} \mathrm{Mater.} \mathrm{Sci.} \mathrm{Eng.} \mathrm{A}$ 682 (2017) 569-576.

[77] S. Ryu, K. Kang, W. Cai, Predicting the dislocation nucleation rate as a function of temperature and stress, J. Mater. Res. 26 (2011) 2335-2354.

[78] Y.J. Wang, M. Zhang, L. Liu, Shigenobu Ogata, L.H. Dai, Universal enthalpyentropy compensation rule for the deformation of metallic glasses, Phys. Rev. B 92 (2015).

[79] A.V. Korchuganov, K.P. Zolnikov, D.S. Kryzhevich, Atomic mechanisms of stacking fault propagation in copper crystallite, Mater. Lett. 252 (2019) 194-197.

[80] S. Mridha, M. Sadeghilaridjani, S. Mukherjee, Activation volume and energy for dislocation nucleation in multi-principal element alloys, Metals (Basel) 9 (2019) 263.

[81] C. Zhu, Z.P. Lu, T.G. Nieh, Incipient plasticity and dislocation nucleation of FeCoCrNiMn high-entropy alloy, Acta Mater. 61 (2013) 2993-3001.

[82] S.P. Wang, J. Xu, Incipient plasticity and activation volume of dislocation nucleation for TiZrNbTaMo high-entropy alloys characterized by nanoindentation, J Mater. Sci. Technol. 35 (2019) 812-816.

[83] A.A. Zbib, D.F. Bahr, Dislocation nucleation and source activation during nanoindentation yield points, Metallurg. Mater. Trans. A 38 (2007) 22492255.

[84] S.K. Lawrence, D.F. Bahr, H.M. Zbib, Crystallographic orientation and indenter radius effects on the onset of plasticity during nanoindentation, J. Mater. Res. 27 (2012) 3058-3065.

[85] A.H.W. Ngan, L. Zuo, P.C. Wo, Size dependence and stochastic nature of yield strength of micron-sized crystals: a case study on Ni3Al. Proceedings of the Royal Society A: mathematical, Phys. Eng. Sci. 462 (2006) 1661-1681.

[86] L.Y. Chen, M.R. He, J. Shin, G. Richter, D.S. Gianola, Measuring surface dislocation nucleation in defect-scarce nanostructures, Nat. Mater 14 (2015) $707-$ 713.

[87] L. Zuo, A.H. Ngan, G.P. Zheng, Size dependence of incipient dislocation plasticity in Ni3Al, Phys. Rev. Lett. 94 (2005) 095501.

[88] A.V. Korchuganov, Atomic-scale mechanisms of single crystal plasticity in $\mathrm{CoCr}$ FeMnNi high-entropy alloys, J. Phys. Conf. Ser. 1147 (2019) 012013.

[89] J.S. Bowles, C.M. Wayman, The bain strain, lattice correspondences, and deformations related to martensitic transformations, Metall. Trans. 3 (1972) 1113-1121.

[90] B. Silvi, A. Savin, Classification of chemical bonds based on topological analysis of electron localization functions, Nature 371 (1994) 683-686.

[91] C.N. Niu, C.R. LaRosa, J.S. Miao, M.J. Mills, M. Ghazisaeidi, Magnetically-driven phase transformation strengthening in high entropy alloys, Nat. Commun. 9 (2018) 1-9.

[92] B.C. Sales, K. Jin, H.B. Bei, G. Stocks, G.D. Samolyuk, A.F. May, M.A. McGuire, Quantum critical behavior in a concentrated ternary solid solution, Sci. Rep. 6 (2016) 26179 . 